\title{
Clots in COVID: A Case Series
}

\author{
Mala V Kaneria ${ }^{1} \odot$, Swaleha Nadaf ${ }^{2}$, Unnati Desai ${ }^{3} \odot$
}

\begin{abstract}
As the novel coronavirus-2019 (COVID-19) pandemic spreads across the world, early recognition of the spectrum of symptoms and patterns of clinical presentation is crucial for optimal management. Emerging evidence shows that COVID-19 leads to a prothrombotic state and its association with pulmonary thromboembolism is well established. However, clinicians and the vascular community, in particular, should watch out for the nonpulmonary targets of this lethal virus as the failure to do so could give rise to disastrous consequences. The significance of raised D-dimer levels, whether it translates into thrombotic events for all patients and the need for universal oral anticoagulation postdischarge are issues that need urgent answers. We report a case series of five patients with thrombosis involving nonpulmonary sites.
\end{abstract}

Keywords: COVID-19, Stroke, Thrombosis.

Indian Journal of Critical Care Medicine (2021): 10.5005/jp-journals-10071-23897

\section{INTRODUCTION}

Since the report of the first COVID-19 cases in Wuhan, China, in December 2019, there is an increasing body of evidence demonstrating thromboembolic events in patients with COVID-19.' Although respiratory failure remains the tipping point and the main cause of mortality in moderate or severe COVID-19, several cardiovascular complications and numerous cases of the thromboembolic disease have been reported. ${ }^{2,3}$

Thrombotic events can occur during COVID-19 as a presenting feature or may occur during convalescence and give rise to lethal consequences.

Severe arterial thrombotic complications have been seen despite the use of antiplatelet and or anticoagulant therapy, in both severe and nonsevere COVID. We report a case series of five patients (two cases with acute onset stroke, two with acute onset lower limb ischemia, and one with cephalic vein thrombosis) who presented with thrombotic complications to our dedicated COVID hospital.

\section{Case Descriptions}

\section{Case 1}

A 50-year-old male, with no existing comorbidities, presented with sudden onset altered sensorium and inability to move the left half of the body. There was no history of fever, vomiting, headache, seizures, diarrhea, or any respiratory complaints. There were no h/o transient ischemic attacks, trauma, or any addictions. As per existing protocol, a nasopharyngeal and oropharyngeal swab (RT-PCR) for COVID 19 was performed at admission, which was positive. The patient had been confined to his home for the past 3 months in view of the lockdown imposed. None of his family members were positive for COVID. On examination, he was drowsy but arousable. He was afebrile, jugular venous pressure not raised, pulse of 90 beats/ minute, regular rhythm, and BP of $160 / 80 \mathrm{~mm} \mathrm{Hg}$. The respiratory rate was $14 /$ minute and the saturation on ambient air was $98 \%$. He moved only the right side on stimulus. There was no neck rigidity and the power was grade 0/5 in the left upper and lower extremity, with the left plantar being extensor. There was a left upper motor neuron facial palsy. The right carotid was feeble to palpate. The rest
${ }^{1}$ Department of Medicine, TNMC and BYL Nair Ch. Hospital, Mumbai, Maharashtra, India

${ }^{2}$ Department of Neurology, TNMC and BYL Nair Ch. Hospital, Nair Hospital, Mumbai, Maharashtra, India

${ }^{3}$ Department of Pulmonary Medicine, TNMC and BYL Nair Ch. Hospital, Mumbai, Maharashtra, India

Corresponding Author: Mala V Kaneria, Department of Medicine, TNMC and BYL Nair Ch. Hospital, Mumbai, Maharashtra, India, Phone: +91 9820210926, e-mail: kaneriamala@rediffmail.com

How to cite this article: Kaneria MV, Nadaf S, Desai U. Clots in COVID: A Case Series. Indian J Crit Care Med 2021;25(7):817-821.

Source of support: Nil

Conflict of interest: None

of the systemic examination including the respiratory and cardiac examinations was unremarkable.

The total counts were $12,100 / \mathrm{mm}^{3}, \mathrm{Hb} 13.4 \mathrm{~g} / \mathrm{dL}$, platelet count $3,02,000 / \mathrm{mm}^{3}$, ESR $100 \mathrm{~mm} /$ hour. Renal and liver function tests and serum electrolytes were within normal limits. Fasting blood sugar was $112 \mathrm{mg} \%$. The $\mathrm{HbA} 1 \mathrm{C}$ was 5.6 and the lipid profile was normal. The inflammatory markers were all elevated viz. $C$ reactive protein $(C R P)>100 \mathrm{mg} / \mathrm{L}$, lactate dehydrogenase (LDH) $898 \mathrm{U} / \mathrm{L}$, and serum ferritin $1254.7 \mathrm{ng} / \mathrm{mL}$. Serum interleukin levels were $>24 \mathrm{pg} / \mathrm{mL}(\mathrm{NR}<7 \mathrm{pg} / \mathrm{mL})$ and D-dimer was $10,000 \mathrm{ng} / \mathrm{mL}(\mathrm{NR}<500)$. Arterial blood gases were normal with a $\mathrm{PaO}_{2} / \mathrm{FiO}_{2}$ ratio $>300$. ECG showed normal sinus rhythm with no e/o left ventricular hypertrophy. 2D echo was normal. HRCT thorax showed bilateral, peripheral rounded ground glass opacities with a CORAD score of $4 / 6$ and 10 to $15 \%$ involvement of the lungs.

CT brain showed a large well-defined intra-axial hypodensity, involving right middle cerebral artery (MCA) territory (subacute infarct) with mass effect in the form of adjacent sulcal and cisternal spaces with ipsilateral lateral ventricle and ipsilateral uncal herniation.

CT brain angiography showed partial filling defect (lumen occluding thrombus) in the right distal common carotid artery

(0) Jaypee Brothers Medical Publishers. 2021 Open Access This article is distributed under the terms of the Creative Commons Attribution 4.0 International License (https://creativecommons.org/licenses/by-nc/4.0/), which permits unrestricted use, distribution, and non-commercial reproduction in any medium, provided you give appropriate credit to the original author(s) and the source, provide a link to the Creative Commons license, and indicate if changes were made. The Creative Commons Public Domain Dedication waiver (http://creativecommons.org/publicdomain/zero/1.0/) applies to the data made available in this article, unless otherwise stated. 
$(4.7 \mathrm{~cm})$ (Fig.1); a complete filling defect in the entire right internal carotid artery and trunk of the external carotid artery (complete lumen occluding thrombus); nonopacification of right MCA with hyperdense MCA sign (Fig. 2).

The patient was treated with intravenous dexamethasone (for raised intracranial tension), subcutaneous low molecular weight heparin $0.6 \mathrm{mg}$ bd, oral ivermectin $12 \mathrm{mg}$ single dose, and vitamins $A, C, D$ with zinc as per existing local COVID treatment guidelines and supportive treatment including physiotherapy. Low-dose aspirin and statin were added to the regimen. His BP and blood sugars were within normal limits throughout the stay. Arterial saturation was maintained on room air throughout the hospital stay.

His subsequent D-dimer after 7 days rose to $25,000 \mathrm{ng} / \mathrm{mL}$ and swab converted to RT-PCR negative after 3 weeks of hospital stay, after which he was discharged on oral rivaroxaban $10 \mathrm{mg} O D$ for 4 weeks.

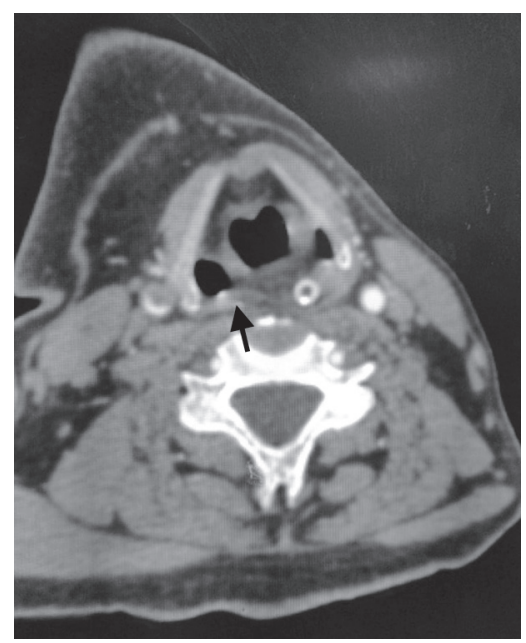

Fig. 1: Partial filling defect (lumen occluding thrombus) in the right distal common carotid artery in Case 1

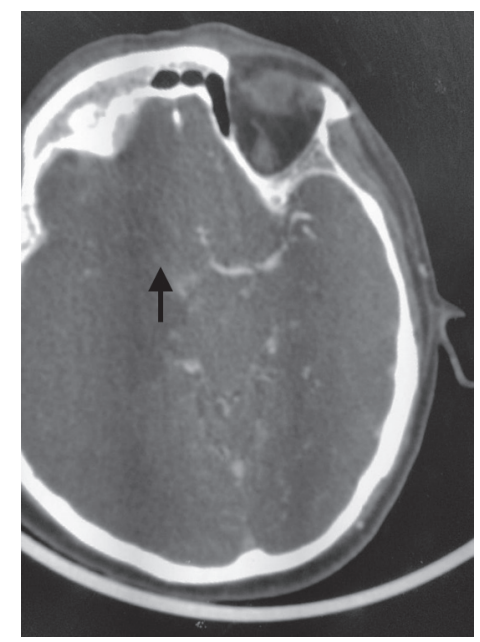

Fig. 2: Nonopacification of the right middle cerebral artery (MCA) with hyperdense MCA sign in Case 1

\section{Case 2}

A 63-year-old male, known hypertensive since 10 years on regular antihypertensives (telmisartan and amlodipine), presented with increasing breathlessness, slurred speech, imbalance, and inability to see objects since 2 days. He gave a h/o fever and minor respiratory symptoms 15 days earlier, when he was diagnosed as COVID on the basis of a positive RT-PCR and also tested positive for vivax on rapid malaria antigen test. He was admitted for isolation in a COVID care center (CCC) and subsequently transferred to our tertiary care institute in view of worsening clinical condition. On examination, his condition was critical, afebrile, BP was 170/86 $\mathrm{mm} \mathrm{Hg}$, pulse $90 /$ minute with a regular rhythm, respiratory rate was $30 /$ minute, and saturation was $88 \%$ on room air. The $\mathrm{PaO}_{2} / \mathrm{FiO}_{2}$ ratio was 250 . He was drowsy but arousable and moving limbs very minimally on stimulus. Chest auscultation revealed bilateral crepitations throughout the lung fields. The rest of the systemic examination was unremarkable. The inflammatory markers were raised viz. ESR $103 \mathrm{~mm} /$ hour, LDH 695 U/L, CRP 156 mg/L, ferritin $2536 \mathrm{ng} / \mathrm{mL}$, and D-dimer was $>15,000 \mathrm{ng} / \mathrm{mL}$. His interleukin 6 level was $>5,500 \mathrm{pg} / \mathrm{mL}$. The rest of the biochemical investigations were normal except for a platelet count of $45,000 / \mathrm{mm}^{3}$. ECG showed borderline left ventricular hypertrophy and $2 \mathrm{D}$ echo was normal.

HRCT thorax performed on admission showed changes s/o COVID with $50-75 \%$ lung involvement. CT brain showed acute infarct in the bilateral occipital lobe, chronic ischemic changes in bilateral frontoparietal, periventricular white matter, lacunar infarcts in bilateral gangliocapsular region (basilar stroke), and corona radiata. There was severe lower and mid basilar cutoff and upper basilar hyperdensity s/o acute progressing thrombus.

He was put on nonrebreathing bag and mask (NRBM) with an oxygen flow of $15 \mathrm{~L}$ and treated with COVID treatment protocol (ivermectin, azithromycin, methylprednisolone, low molecular weight heparin) antimalarials, and supportive care. Tocilizumab, an interleukin 6 receptor antagonist, was also given in view of the cytokine storm. His condition deteriorated on day 2 of admission and he succumbed.

\section{Case 3}

A 63-year-old male, k/c/o diabetes and hypertension, presented with increasing breathlessness since 7 days and right lower limb pain and discoloration of toes. He had minor respiratory complaints 15 days ago for which he took symptomatic treatment. He tested positive for COVID RT-PCR. There were no other major complaints. On examination, he was stable, afebrile with a respiratory rate of 14 /minute, BP of 130/80 $\mathrm{mm} \mathrm{Hg}$, absent right dorsalis pedis, and posterior tibial pulsations. There was bluish discoloration of toes on the right lower extremity. All other peripheral pulsations were well felt. His saturation was $92 \%$ on room air, which increased to $98 \%$ on $4 \mathrm{~L}$ oxygen by nasal prongs. The rest of the systemic examination was normal.

His inflammatory markers (CRP, LDH, ferritin, ESR) were all elevated. D-dimer was $>15,000 \mathrm{ng} / \mathrm{mL}$. Interleukin 6 levels were $5337.6 \mathrm{pg} / \mathrm{mL}$. Prothrombin time, prothrombin index, and INR were all normal. Hemogram and rest of the biochemical parameters were normal. HRCT thorax showed $50-60 \%$ lung involvement (CORADS 6). ECG and 2D echo were within normal limits. USG Doppler revealed the absence of color flow in right anterior tibial (ATA), posterior tibial (PTA), and dorsalis pedis arteries (DPA). CT angiography of lower extremities showed atherocalcific changes in distal abdominal aorta and bilateral common iliac arteries 
not causing significant luminal obstruction and thrombus in the abdominal aorta (Fig. 3). Nonopacification of proximal two-third of left superficial femoral artery, distal one-third of ATA, right tibioperoneal trunk, right anterior and posterior arteries in the entire extent, bilateral PTAs s/o complete lumen occluding thrombosis.

He was treated with oxygen by nasal prongs, intravenous methylprednisolone, low molecular weight heparin, ivermectin, doxycycline, and vitamins. Low-dose aspirin, statin, cilostazol, and pentoxifylline were also added. Blood sugars were monitored and controlled with insulin. Conservative treatment was advised by the cardiovascular thoracic surgeon. The patient gradually recovered and was discharged after around 45 days when the swab converted to negative. Oral rivaroxaban $10 \mathrm{mg}$ OD was added for 1 month as his predischarge D-dimer was significantly raised.

\section{Case 4}

A 53-year-old male, k/c/o diabetes and hypertension, presented with increasing breathlessness since 2 days and bluish discoloration of all the phalanges of the left lower limb (Fig. 4). He was admitted with mild respiratory symptoms and COVID positive status at another hospital 20 days ago, treated, and subsequently discharged from there. On examination, he was afebrile, tachypnoeic with a respiratory rate of $40 /$ minute, saturation of $80 \%$ on room air, and hemodynamically stable. His left lower extremity was cold with the absence of left femoral,

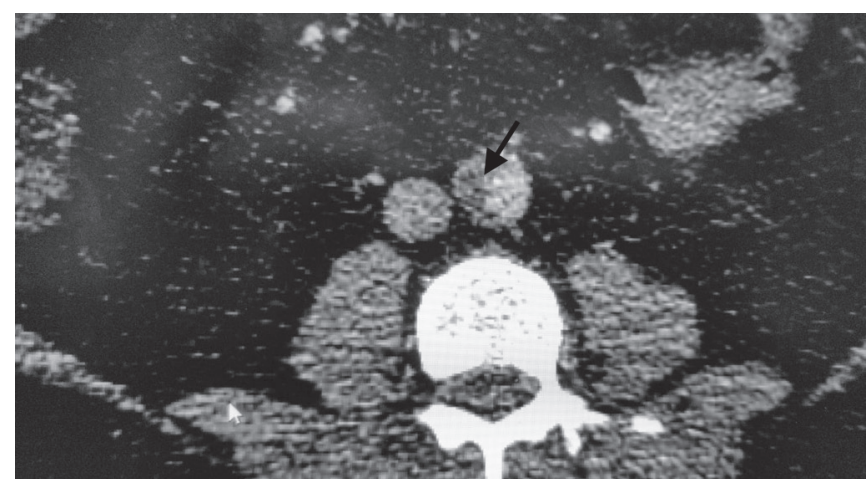

Fig. 3: Thrombus in the abdominal aorta of Case 3

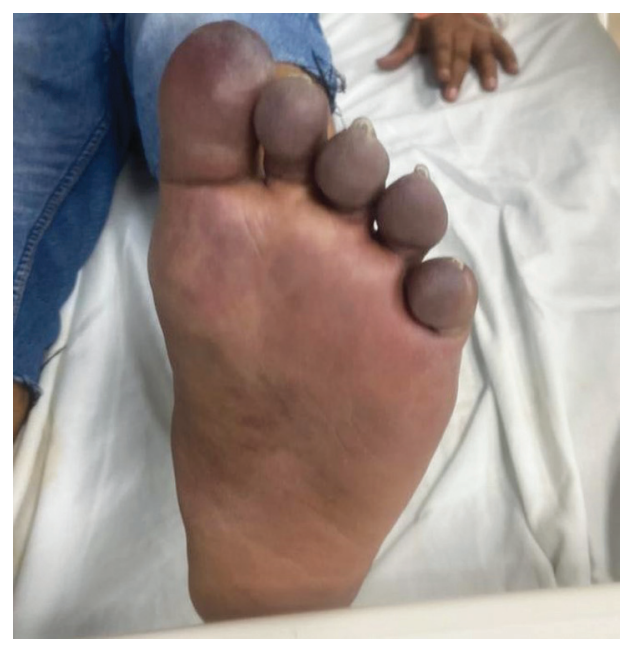

Fig. 4: Bluish discoloration of the toes in Case 4 left tibial, and left dorsalis pedis pulsations. His CT angiography of the lower limbs showed a complete lumen occluding thrombus in the left popliteal artery, distal to the knee joint (Fig. 5). HRCT thorax showed COVID pneumonia with 50-75\% lung involvement. His blood investigations revealed a leukocytosis of $44,500 / \mathrm{mm}^{3}$, INR of 1.55 , and D-dimer $>20,000 \mathrm{ng} / \mathrm{mL}$. The inflammatory markers were all raised. He was started on BiPAP with a $\mathrm{FiO}_{2}$ of $85 \%$. Conservative treatment was decided by a multidisciplinary team considering the severity of respiratory distress. However, he succumbed shortly after admission.

\section{Case 5}

A 25-year-old female, a frontline healthcare worker with no comorbidities, presented with fever and mild respiratory symptoms of 5 days duration. She tested positive for COVID RT-PCR and was hospitalized. She was stable and her general and systemic examination was normal, except for fever. Saturation on room air was $98 \%$. HRCT thorax showed $<10 \%$ involvement of the lungs. Her CRP, ferritin, LDH, and ESR were slightly raised and D-dimer was $>15,000$. The rest of the biochemical parameters were normal except for a total count of $16,500 / \mathrm{mm}^{3}$. She was treated with ivermectin, Doxy, low molecular weight heparin, and vitamins. Intravenous ceftriaxone was given for a day in view of fever and leukocytosis but was stopped the next day as her fever subsided, counts declined, and the serum procalcitonin was normal. On day 10 , at the time of discharge, she complained of pain and mild swelling of the right hand, just above the wrist. A USG Doppler showed a $3.7 \mathrm{~cm}$ thrombus in the right cephalic vein.

\section{Discussion}

The pathogenesis of COVID-19 is insidiously unfolding. It is now being considered a prothrombotic disease believed to be responsible for venous and arterial thrombosis. Many patients with severe COVID-19 present with coagulation abnormalities that mimic other systemic coagulopathies, but the significance of COVID-19 coagulation abnormalities is becoming increasingly clear as a sizeable proportion of patients with severe COVID-19 develop, sometimes unrecognized, venous and arterial thromboembolic complications. ${ }^{4}$ It is believed that COVID-19 coagulopathy might be a form of disseminated intravascular coagulation. ${ }^{5}$ Acute arterial occlusions have been described in the aorta and mesenteric and cerebral arteries. ${ }^{6,7}$

The prevalence of thrombosis in COVID-19 is multifactorial: the presence of a raging inflammatory response accompanied by coagulopathy, with raised D-dimer levels, and the frequent presence of antiphospholipid antibodies; ${ }^{8}$ and a higher risk for complications, such as atrial arrhythmias, myocardial infarction, heart failure, myocarditis, and venous thromboses, which contribute to the risk of ischemic stroke. ${ }^{9}$ Additionally, baseline stroke risk factors, such as hypertension, diabetes, and coronary artery disease, predispose a patient to infection with COVID-19.

The most classical feature in patients presenting with thrombotic complications in COVID-19 is an increased D-dimer and a modest thrombocytopenia. ${ }^{10}$ All our patients had elevated D-dimer levels and developed thrombosis in spite of being on adequate and appropriate anticoagulation, which is concurrent with the observation of others. ${ }^{11}$ Low platelet count was observed only in the patient with coexisting malaria. It has been reported that about $50 \%$ of the patients have increased D-dimer levels, and abnormal D-dimer levels are associated with a poor prognosis. ${ }^{12}$ The diagnostic value of D-dimer levels for thrombus formation in COVID-19 patients is unclear. Whether 


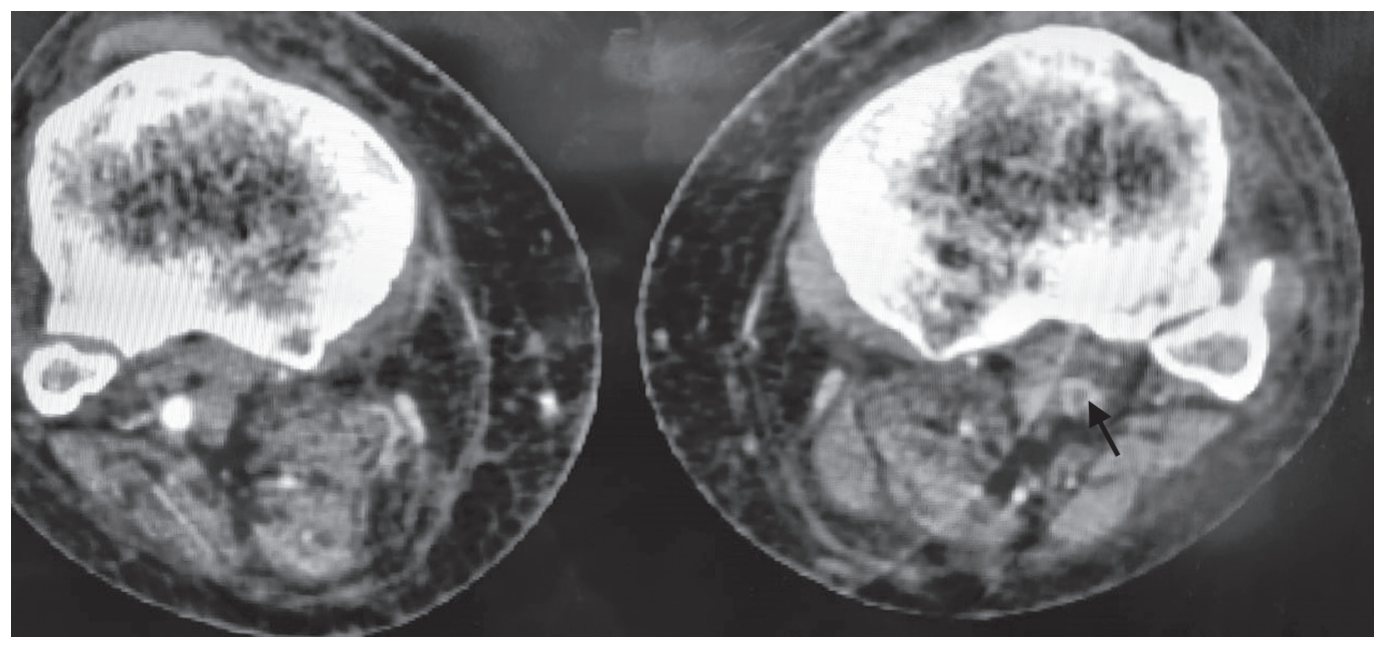

Fig. 5: Lumen occluding thrombus in the left popliteal artery in Case 4

higher D-dimer levels in patients with COVID-19 suggest the need for more aggressive anticoagulant therapy is a matter of debate. ${ }^{12}$ Three of our five patients survived and were discharged after a prolonged stay. Two of our patients succumbed, one due to a stormy pulmonary course and the second due to pulmonary and neurological complications.

Majority of the cases described in the literature had pre-existing comorbidities, which could have contributed to the thrombotic complication. ${ }^{13}$ However, two of our five patients did not have the traditional risk factors predisposing them to thrombosis or arterial embolisms (Cases 1 and 5) and one was a well-controlled hypertensive (Case 2). In some series, thrombotic manifestations have been described in the elderly, though none of our patients could be categorized as elderly.

Most of the reports have described thrombosis in patients with severe COVID. ${ }^{1,14}$ In our case series, two patients (Cases 1 and 5) had mild-COVID and did not need supplemental oxygen. In fact, the first patient was probably asymptomatic and was diagnosed as COVID when he presented with a stroke.

Acute ischemia/thrombosis appeared on average 15 days after the onset of respiratory COVID-19 symptoms, in most of the cases reported in the literature. In the study of Mao et al., $5.7 \%$ of patients with severe infection developed cerebrovascular disease later in the course of illness. ${ }^{13}$ In our study, patients 2 and 4 had severe respiratory involvement at the time of presentation with the thrombotic complication, though the day of symptom onset was 15-20 days earlier.

CRP and ferritin were elevated in all the patients, implying a role of inflammation, which has been increasingly accepted as a contributor to the thrombotic events. ${ }^{15}$ Elevated levels of CRP and D-dimer, indicating a high inflammatory state and abnormalities with the coagulation cascade, respectively, might play a role in the pathophysiology of stroke in the setting of COVID-19 infection. ${ }^{13}$

Two of the patients (Cases 2 and 4) were treated for mild COVID symptoms and developed life-threatening thrombotic complications after discharge. In view of the severe respiratory involvement and the poor general condition of both the patients, intervention could not be offered to them.

In view of the relative recency of the pandemic and current limited evidence, the role of oral anticoagulation for all patients postdischarge is not clear. In addition, D-dimer levels rise after the second week in many patients, at all levels of severity. Also, at what level of $\mathrm{D}$-dimer should oral anticoagulation be initiated and for how long is a matter of debate. The benefit of short-term oral anticoagulation at the time of discharge, preferably based on predischarge D-dimer levels, needs to be further evaluated.

\section{Conclusion}

Catastrophic COVID complications, such as acute ischemic stroke and lower limb arterial thrombosis, should be picked up early so that time-sensitive interventions, such as thrombolysis and thrombectomy, can be instituted in order to avoid longterm disability. Stroke teams should be aware of the fact that COVID-19 patients can present with cerebrovascular accidents and use appropriate personal protective equipment in every suspected patient. It is well established that all hospitalized patients with COVID-19 should receive prophylactic or therapeutic anticoagulation. But what needs to be elucidated through trials is the benefit of short-term anticoagulation postdischarge, its duration, and the most appropriate agent for the same.

Meanwhile, unraveling thrombotic mechanisms in COVID- 19 may yield better strategies to prevent disabling thrombotic complications, like ischemic stroke and limb ischemia.

\section{OrCID}

Mala V Kaneria 나 https://orcid.org/0000-0003-0115-0855

Swaleha Nadaf ㄴ https://orcid.org/0000-0002-8953-9875

Unnati Desai $\odot$ https://orcid.org/0000-0002-7647-0469

\section{References}

1. Terpos E, Ntanasis-Stathopoulos I, Elalamy I, Kastritis E, Sergentanis TN, Politou M, et al. Hematological findings and complications of COVID-19. Am J Hematol 2020. DOI: 10.1002/ajh.25829.

2. Wang D, Hu B, Hu C, Zhu F, Liu X, Zhang J, et al. Clinical characteristics of 138 hospitalized patients with 2019 novel coronavirus-infected pneumonia in Wuhan, China. JAMA 2020;323(11):1061-1069. DOI: 10.1001/jama.2020.1585.

3. Tang N, Bai H, Chen X, Gong J, Li D, Sun Z. Anticoagulant treatment is associated with decreased mortality in severe coronavirus disease 2019 patients with coagulopathy. JThromb Haemost 2020;18(5):10941099. DOI: 10.1111/jth.14817. 
4. Thachil J, Tang N, Gando S, Falanga A, Cattaneo M, Levi M, et al. ISTH interim guidance on recognition and management of coagulopathy in COVID-19. J Thromb Haemost 2020;8(5):1023-1026. DOI: 10.1111/ jth. 14810

5. Tang N, Li D, Wang X, Sun Z. Abnormal coagulation parameters are associated with poor prognosis in patients with novel coronavirus pneumonia. J Thromb Haemost 2020;18(4):844-847. DOI: 10.1111/ jth.14768.

6. Le Berre A, Marteau V, Emmerich J, Zins M. Concomitant acute aortic thrombosis and pulmonary embolism complicating COVID-19 pneumonia. Diagn Interv Imaging 2020;101(5):321-322. DOI: 10.1016/j.diii.2020.04.003.

7. de Barry O, Mekki A, Diffre C, Seror M, Hajjam ME, Carlier RY. Arterial and venous abdominal thrombosis in a 79-year-old woman with COVID-19 pneumonia. Radiol Case Rep 2020;15(7):1054-1057. DOI: 10.1016/j.radcr.2020.04.055.

8. Zhang Y, Xiao M, Zhang S, Xia P, Cao W, Jiang W, et al. Coagulopathy and antiphospholipid antibodies in patients with COVID-19. N Engl J Med 2020;382(17):e38. DOI: 10.1056/NEJMc2007575.

9. Goyal P, Choi JJ, Pinheiro LC, Schenck EJ, Chen R, Jabri A, et al. Clinical characteristics of COVID-19 in New York City. N Engl J Med 2020;382(24):2372-2374. DOI: 10.1056/NEJMc2010419.
10. Guan WJ, Ni ZY, Hu Y, Liang WH, Ou CQ, He JX, et al. Clinical characteristics of coronavirus disease 2019 in China. N Engl J Med 2020;382:1708-1720. DOI: 10.1056/NEJMoa2002032.

11. Kashi M, Jacquin A, Dakhil B, Zaimi R, Mahé E, Tella E, et al. Severe arterial thrombosis associated with Covid-19 infection. Thromb Res 2020;192:75-77. DOI: 10.1016/j.thromres.2020.05.025. PMCID: PMC7229939. PMID: 32425264.

12. Yu B, Li X, Chen J, Ouyang M, Zhang H, Zhao X, et al. Evaluation of variation in $D$ dimer levels among COVID-19 bacterial pneumonia: a retrospective analysis. J Thromb Thrombolysis 2020;50(3):548557. DOI: 10.1007/s11239-020-02171-y. PMCID: PMC7286212. PMID: 32524516.

13. Avula A, Nalleballe K, Narula N, Sapozhnikov S, Dandu V, Toom S, et al. COVID 19 presenting as stroke. Brain Behav Immun 2020;87:115-119. DOI: 10.1016/j.bbi.2020.04.077. PMCID: PMC7187846. PMID: 32360439.

14. Bhatraju PK, Ghassemieh BJ, Nichols M, Kim R, Jerome KR, Nalla AK, et al. Covid-19 in critically ill patients in the Seattle region - case series. N Engl J Med 2020;382(21):2012-2022. DOI: 10.1056/NEJMoa2004500.

15. Klok FA, Kruip MJHA, van der Meer NJM, Arbous MS, Gommers DAMPJ, Kant KM, et al. Incidence of thrombotic complications in critically ill ICU patients with COVID-19. Thromb Res 2020;S00493848(20)30120-1. DOI: 10.1016/j.thromres.2020.04.013. 